EVEL YN L. BARBEE

\title{
FEELING OLDER AND WANTING TO BE YOUNGER*
}

\begin{abstract}
Two samples of middle-aged women were compared using a questionnaire adapted from Rossi (1980). One sample $(N=20)$ consisted of women who felt older than and desired to be younger than their own age. The second sample $(N=19)$ was comprised of women who felt and desired to be their own age. Statistically significant differences between the groups were that women who felt older reported more physical changes of aging, less satisfaction with their life context, and more worries than the women who felt their chronological age. Results of this study suggest that current life stress contributes to middle-aged women's perceptions of feeling older and desiring to be younger than their own age.
\end{abstract}

Key Words: middle-aged women, aging, perceptions of aging, life context and aging, physical changes of aging.

\section{INTRODUCTION}

Although diversity among the elderly is generally recognized, our knowledge about the variability among middle-aged women is limited. This is especially true in regard to variability among low-income, middle-aged women. A major reason for the paucity of knowledge about middle-aged women is that most of the research concerned with middle-aged persons has used men as subjects (cf. Levinson, Darrow, Klein, Levinson, and McKee 1978; Vaillant 1977). Another reason for the lack of knowledge about middle-aged women is professional short sightedness. Because professionals have tended to use a one dimensional approach to the study of middle-aged women, much of the literature on this group focuses on menopause or menopausal symptoms. The net result is that the concentration in terms of middle-aged women has been related to a biological change associated with reproduction. Since these topics are not the subject of this paper this large body of literature is not reviewed.

The purpose of this paper is to compare the responses of two groups of middle-aged women (one of which feels and desires to be their own age and the other of which feels older and desires to be younger than their chronological age) for selected variables. The findings presented in this paper are part of a larger, exploratory, cross-sectional, comparative study that explored life experiences and aging in a group of predominantly low income, middle-aged African American and white women. It is suggested here that the cross-cultural study of variability in adult development can be enhanced through the use of concepts from anthropology, sociology and psychology. 


\section{THEORETICAL PERSPECTIVES}

\section{Perspectives on Age}

As Fry (1980) points out, anthropology's historical interest in age has not been accompanied by an equally strong interest in aging. Basically age has been investigated as a principle of social organization (Fry 1980) with the view that age is incorporated into social structure by way of the individual life cycle (Fortes 1984). Anthropology's recognition of the aged as a group and increased interest in age as a principle for corporate organization, social, economic and political demands (Simic 1978), is evidenced by the recent organization of the Association of Anthropology and Gerontology.

As basic components of socio-cultural life, there are several aspects of age and the aging process that contribute to their being essential ingredients of all cultures. First, as a cultural domain, age is an ascribed characteristic of all individuals (Fry 1980). Second, if one lives long enough, that is if the process of aging goes on long enough, age becomes an achieved characteristic. Third, the process of aging and age are incorporated, elaborated upon and interpreted by a complex world of symbols (Fry 1980). Fourth, as Goody (1976: 117) reminds us, the process of aging influences all types of social groups and "every type of social relationship." Age and aging are both observable and experiencecd and are invested with significant cultural value (Fortes 1984).

Researchers disagree as to whether chronological age is an important index in research with middle-aged persons. Neugarten and Datan (1974) suggest that chronological age is not a meaningful gauge when studying middle adulthood because the major life events that characterize the middle years, e.g., grandparenthood, menopause, the death of parents, etc., may occur at different chronological ages and may be separated by different intervals of time. Neugarten and Datan (1974) suggest that middle age may be a state of mind that affects individuals' perceptions of themselves and their strategies for managing their worlds.

In commenting on the paucity of cross-cultural research on middleaged women, Brown (1982) suggests that this state of affairs is due both to a lack of appropriate ethnographic data and difficulties in defining middle age. Brown (1982: 143) defines middle-aged women ". . . as mothers who are not yet aged but who have adult offspring." Similarly, Rubin (1979) suggests that mid-life begins for women when their children are grown. There are two major problems with both Rubin's (1979) and Brown's (1982) definitions of middle age. First, these definitions exclude women who are not mothers. Second, because large numbers of women in Western societies delay childbearing, the range of childbearing years is wider. As a result, the age range for mid-life women would also be quite wide. 
Fortes (1984: 101) states that in Western societies the norms of chronological age are applied to maturational stages because, "... the apparatus is available and dominates our thinking about maturation and because the laws that define and determine the rights and duties of citizenship demand it." In exploring the age forty, Brandes (1985) concludes that cultural reasons underlie our perceptions of this age as a transitional period. Since chronological age is symbolically linked with important rules in this society (e.g., when children start school, when one can retire), the importance of chronological age and one's perceptions of one's chronological age should not be underestimated.

\section{Perspectives on Middle-Aged Women}

The aging of middle-aged women is usually viewed in a biased way by myths and stereotypes. Thus, in the United States it is popularly assumed that middle-aged women's desires to be younger than their chronological age are based upon subscription to the socio-cultural norm of youthfulness. For example, Henry (1973) writes about "forty-year-old jitters" in middle-aged women and suggests that one of the cultural sources for problems in middle-aged women in the United States is an emphasis on youth, beauty and romantic love. Although Henry's (1973) conclusions are based upon an analysis of the general features of United States urban social organization, his analysis does not deal with the more immediate context of women's lives.

Much of the literature on middle-aged women's development critiques the use of stage theories ${ }^{1}$ with women. For example, Barnett and Baruch (1978) point out that the normative life crisis model focus on chronological age and linear stages of development reflect male experience. In commenting upon the inadequacy of the stage model of development, Steitz (1981-82) points out that the increased pace and diversity of sociocultural change makes chronological age inadequate as the sole basis for interpreting developmental events.

Brown (1982) uses a framework based upon sociobiology, psychoanalytic theory, systems of inheritance, relations between spouses, parenting behavior and species survival to explore the status change of middleaged women and the relationships between middle-aged women and their adult children. From a selective review of the ethnographic literature, Brown (1982: 143) concludes that for women outside of Western society: "Middle-age brings fewer restrictions, the right to exercise authority over certain kinsmen, and the opportunity for achievement and recognition beyond the household." While Brown's (1982) assertion that certain ascriptions prevalent toward middle-aged women in Western society (e.g., "forty-year-old jitters," "empty nest" and "rolelessness") do not apply cross-culturally, the relevance of these assertions for all middle-aged 
women in Western society can also be questioned. For example, Bart (1971) found that women who chose the traditional, non-aggressive, childcentered, feminine role are most prone to the "empty nest" syndrome when their children leave home.

Rubin (1979) while dealing with working class middle-aged women, focuses on role conflicts and role alterations. Brown's (1982) and Brown's and Kerns' (1985) concerns are with examining and enlarging crosscultural research with middle-aged women not with their perceptions of aging. Although Datan, Antonovsky and Maoz (1981) did cross-cultural research with middle-aged women, their primary focus was on psychological responses to the menopause.

As Rossi (1980) points out the immediate context of middle-aged women's lives includes physical changes of aging, worries about their family income, worries about their marriage, and degree of satisfaction with major aspects of their lives. Alston and Rose (1981) in a study of middle-aged African American women's perceptions of aging suggested that the women rejected the typical ideas about physical appearance at middle-age. Rossi (1980) in a study of 64 middle-aged, middle to upper income women concluded that women under stress desired to be younger than their chronological age. However, Rossi (1980) did not compare women who desired to be younger than their chronological age with those who desired to be their own age. An understanding of women's experience and the affects of these experiences on their perceptions of aging will assist us in comprehending not only why some women desire to be younger than their chronological age but also why they feel older than their actual age.

Kertzer and Keith (1984) believe that the challenge to social scientists today involves contributing to one's own discipline while simultaneously broadening it through the use of pertinent new concepts developing in other disciplines. Two concepts that are particularly pertinent to understanding middle-aged women's perceptions of their own aging are the sociological perspective of the life course and psychology's dialectical approach to aging. The life course perspective, ${ }^{2}$ a sociological perspective on aging, is concerned with the demographic concept of cohort analysis, that is, charting the historical context in which subjects live out their adult lives (Riley and Abeles 1982).

Two principles from the life course perspective which are relevant to the research reported here are: (1) aging is influenced by the society in which people live; that is within the same society different groups female and male, rich and poor age in different ways and (2) that of cohort (Riley and Abeles 1982). Cohort analysis in some ways is similar to the anthropological concept of age-grade. Age-grades are cultural categories of the life course. Thus, there are a set of age categories through which people must pass and in which their status is formally defined. Movement from one category to another may involve collective transitions. 
The dialectical perspective represents a rejection of the ontogenetic assumptions of the stage model of development (Dannefer 1984). The dialectical approach to adult development is primarily identified with Riegel $(1975,1976,1979)$. Riegel was primarily interested in the developmental interactions between and among the individual, the social group, and the environment. The dialectical theory of adult development focuses on movement or progression along four, mutually independent, interactive dimensions. These dimensions are (1) inner-biological, (2) individualpsychological, (3) cultural-sociological, and (4) outer-environment.

The inner biological dimension includes: physical maturation (which includes changes due to aging), illness, accidents, and, death (Riegel 1975, 1976, 1979). Interpersonal relationships between and among parents and children, spouses, and siblings, are included in the individual psychological dimension (Riegel 1975, 1976, 1979). The intrapersonal aspects of the individual psychological dimension are represented by the inner dialogue, doubts, and questions that are the foundation of thought and action (Riegel 1979). Some of the concerns within the cultural-sociological dimension are institutions, laws, norms and traditions regarding marriage and childbirth (Riegel 1975, 1976, 1979). The outer-physical dimension includes events such as natural and human disasters (wars, earthquakes, drought), and structural and economic conditions (Riegel 1975, 1976, 1979).

Asynchrony within, between, or among these dimensions is caused by contradictions (Riegel 1975, 1979). These contradictory conditions are the fundamental basis for development and change because they produce constructive crisis (Riegel 1975, 1976, 1979). The crisis that results from asynchrony produces a new level of adult development. In contrast to the stage theories of development, a dialectical interpretation of development and change does not emphasize stages or plateaus at which equilibrium is achieved. Stable plateaus of balance, stability, and equilibrium occur when a developmental or historical task is completed.

The dialectical paradigm is interested in both short term situational changes as well as with long term socio-cultural and individual developments. As a paradigm for adult development, the dialectical perspective is concerned with raising questions and seeking answers to individuals growing and developing together.

\section{RESEARCH PURPOSES AND QUESTIONS}

The purpose of this research was to examine desiring to be younger than and feeling older than chronological age in a group of middle-aged women. A second purpose was to compare commonalities and differences between women who wanted to be younger and felt older than their chronological age and women who felt and desired to be their actual age. 
The research described in this paper is part of a larger, exploratory, cross sectional, research project that examined life experiences and perceptions of aging in middle-aged African American and white women. The portion described here asked the following question:

Do women who feel older than and want to be younger than their chronological age differ on the variables of interest in this study from women who wish to be and feel their chronological age?

METHOD

\section{Subjects}

The research population for the larger study consisted of 207 (107 African American and 100 White) mothers, between 32 to 56 years of age. This was a convenience sample. Sample criteria were mothers between thirty-two and fifty six years of age. Women who met the sample criteria came from three ambulatory care health centers in a large, Midwestern metropolitan area.

A major disadvantage in using a clinic population in a study that includes questions about health is that the results may be confounded by manifest health status. However, only $30 \%$ of the older African American, $6 \%$ of the younger African American, and $4 \%$ of the older white women were seeking care for themselves. Forty-one percent of the total sample were seeking health care for their children or grandchildren, $33 \%$ accompanied friends, $15 \%$ were employed at the clinics and $11 \%$ were seeking health care for themselves. A major advantage of using a clinic population was that it allowed access to a population of predominantly low-income women, a group not traditionally used in research on the middle-aged.

Two sub-samples were drawn from the larger population. The first subsample, Aged 1, consisted of nineteen women (10 African American and nine white) who felt and desired to be their own age. The second subsample, Aged 2, consised of twenty women (12 African American and 8 white) who felt older than and desired to be younger than their chronological age.

\section{Apparatus}

An interview protocol was adapted from Rossi (1980). The interview protocol included demographic questions, questions about aging signs and symptoms, satisfactions, worries and open-ended questions about age perceptions. Each woman was interviewed for twenty-five minutes.

The variables used in this research were satisfactions, aging signs and 
symptoms, worries, perceptions of current life and age perceptions. In addition, the women were asked open-ended questions about what were the best and worst things about being their age. Aging signs and symptoms were measured as:

(1) A three point rating of "better five years ago," "no change" or "better now" of health, eyesight, hearing, energy level, shape of body, sex life, hair condition, weight and teeth.

Satisfactions were measured by:

(2) A five point rating from very dissatisfied to very satisfied on seven items; house or apartment, neighborhood, social life, relationship with husband, relationship with children, relationship with boyfriend and time to self.

Worries were measured by:

(3) A three point rating from "not at all" to "great deal" of family income, your health, husband's health, children's health, your marriage, your relationship with children, husband's relationship with children, and thinking about your own future as sources of worry.

Perceptions of current life were measured by responses to "Which best describes the past five years of your life?":

(1) The very best years of my life.

(2) Among the best years of my life.

(3) No difference from any other years of my life.

(4) Among the worst years of my life.

(5) The very worst years of my life.

The age perceptions were measured as:

(1) Subjective age - answers to the question "How old do you feel most of the time?"

(2) Desired age, answers to the question. "If you could be any age you wanted to be right now, what age would you like to be?"

(3) Desired death age - Age at which the women desired to die.

(4) Expected death age - Age at which the women expected to die.

Since the interest was not to measure the above variables over time but to identify current responses, test-retest was not used for reliability.

\section{RESULTS AND DISCUSSION}

Chi square tests were used to compare the responses of both groups on the aging signs and symptoms index, worries and satisfactions. The significance level was set at 0.05 . The Aged 1 group consisted of the women who felt and desired to be their own age. The Aged 2 group consisted of women who felt older and desired to be younger than their chronological age.

Table I displays the demographic data on the groups. The two groups 
TABLE I

Demographic data

\begin{tabular}{|c|c|c|}
\hline & \multicolumn{2}{|l|}{ Group } \\
\hline & $\begin{array}{l}\text { Aged } 1 \\
N=19\end{array}$ & $\begin{array}{l}\text { Aged 2 } \\
N=20\end{array}$ \\
\hline \multicolumn{3}{|l|}{ Marital Status } \\
\hline Married & 47.0 & 25.0 \\
\hline Separated & 5.0 & 25.0 \\
\hline Divorced & 32.0 & 35.0 \\
\hline Widowed & 11.0 & 5.0 \\
\hline Never Married & 5.0 & 10.0 \\
\hline Mean Age & 42.0 & 41.0 \\
\hline Mean Number of Children & 4.0 & 3.0 \\
\hline Mean Number of Children at Home & 2.0 & 2.0 \\
\hline \multicolumn{3}{|l|}{ Employment } \\
\hline Employed & 37.0 & 30.0 \\
\hline Not employed & 63.0 & 70.0 \\
\hline Husband employed & 57.0 & 40.0 \\
\hline \multicolumn{3}{|l|}{ Education } \\
\hline Grade school or less & 16.0 & 5.0 \\
\hline Some high school & 42.0 & 20.0 \\
\hline High school graduate & 16.0 & 55.0 \\
\hline Some college & 26.0 & 20.0 \\
\hline College graduate or more & 10.0 & - \\
\hline \multicolumn{3}{|l|}{ Income } \\
\hline Under 4,999 & 29.0 & 40.0 \\
\hline $5,000-9,999$ & 6.0 & 20.0 \\
\hline $10,000-14,999$ & 18.0 & 10.0 \\
\hline $15,000-19,999$ & 12.0 & 5.0 \\
\hline $20,000-24,999$ & 12.0 & 15.0 \\
\hline Over 25,000 & 24.0 & 10.0 \\
\hline
\end{tabular}

differed in several important demographic aspects. In terms of marital status, more women from Aged 1 were married $(47 \%)$ than women in Aged $2(25 \%)$. The women in the Aged 2 group reported a generally higher education level (55\%) completed high school versus $16 \%$ in the Aged 1 group. The income of the Aged 2 group was not reflected by their educational level.

Although forty per cent of Aged 2 reported family incomes of less than $\$ 4,999$ a year compared with $29 \%$ of the Aged 1 group, the differences in their incomes were not statistically significant. Only $30 \%$ of the Aged 2 group was employed versus $37 \%$ of the Aged 1 group. The difference in income between the two groups was probably due to three reasons; unemployment, concentration in lower paying employment, and fewer marriage partners for the Aged 2 group. The Aged 1 group reported 
having a higher mean number of children than the aged 2 group. Both groups reported have the same average number of children at home, two.

There were two significant differences between the groups on the aging signs and symptoms index. More women from Aged 2 group reported that their energy level was better five years ago $\chi^{2}(2, N=39)=10.99, p<$ 0.01 . This finding is somewhat similar to those of Alston and Rose (1981) who reported that the women in their study "tired easily." Also more women from Aged $2 \chi^{2}(2, N=39)=6.21, p<0.05$ reported that their hair condition was better five years ago.

There were significant differences between the two groups on four of the seven areas of satisfaction. The Aged 2 group reported more mixed feelings to dissatisfaction than the Aged 1 with their social life, $\chi^{2}(1, N=$ $39)=9.29, p=0.01$; their neighborhood, $\chi^{2}(1, N=39)=4.21, p=$ 0.02 ; their relationship with their children, $\chi^{2}(1, N=39)=6.78, p<$ 0.01 ; and their relationship with their husbands, Fisher's Exact Test, $N=$ 15, $p=0.01$. In addition, the women in Aged 2 reported more worries about their family income, $\chi^{2}(2, N=39)=9.11, p=0.01$ and their marriage, Fisher's Exact Text, $N=14, p=0.03$.

An examination of Table II demonstrates that on the age perception variables that there were no commonalities between the groups. The women in group Aged 1 were an average of twenty years of age when they gave birth to their first child. The average age of Aged 2 group when they gave birth to their first child was tweny-one years. Since the two groups were drawn because of their responses to the age perception questions, there were expected differences on these variables, particularly in terms of desired and subjective ages.

TABLE II

Age perceptions by group

\begin{tabular}{lll}
\hline & \multicolumn{1}{l}{ Group } & \\
\cline { 2 - 3 } & $\begin{array}{l}\text { Aged 1 } \\
(N=19)\end{array}$ & $\begin{array}{l}\text { Aged 2 } \\
(N=20)\end{array}$ \\
\hline $\begin{array}{l}\text { Desired Age } \\
\text { Mean }\end{array}$ & 42.0 & 19.0 \\
$\begin{array}{l}\text { Subjective Age } \\
\text { Mean }\end{array}$ & 42.0 & 54.0 \\
$\begin{array}{l}\text { Desired Death Age } \\
\text { Median } \\
\quad \text { Range }\end{array}$ & 90.0 & 83 \\
$\begin{array}{l}\text { Expected Death Age } \\
\text { Median } \\
\text { Range }\end{array}$ & $(65-115)$ & $(70-200)$ \\
\hline
\end{tabular}


The respective mean chronological ages of Aged 1 and Aged 2 groups were 42.0 and 41.0 years of age. In terms of desired age, Aged 2 desired to be a mean of twenty-two years younger than their mean chronological age. Interestingly enough, their average desired age was younger than the average age at which they gave birth to their first child. In terms of their subjective age, the Aged 2 group reported feeling a mean of 13 years older than their mean chronological age.

Both groups expressed a desire to live a long life. The Aged 1 group reported a median desired death age that was seven years higher than that of Aged 2. In a similar vein, the Aged 2 group reported that they expected to live a median that was four years less ( 70 years versus 74 years) than that of the Aged 1 group.

When asked about the best thing about being their current age, the most frequent response for both groups was "knowledge, experience and maturity." The respective percentages of Aged 1 and Aged 2 reporting knowledge, experience and maturity were $53 \%$ and $35 \%$. Twenty percent of the Aged 2 group said nothing was good about being their age. None of the Aged 1 group responded "nothing."

In terms of the worst thing about being their age, the most frequent responses for both groups were related to physical changes of aging. Thirty-seven percent of Aged 1 and $50 \%$ of the Aged 2 reported that physical changes were the worst thing about being their age. When asked about the past five years of their life a major difference between the groups emerged. Sixty-eight percent of the Aged 1 group reported that the past five years were "the best" to among the "very best" years. Only $21 \%$ of the Aged 2 group responded that the past five years were among the best.

The findings for the Aged 2 group suggest that women who desire to be younger than and feel older than their chronological age are not just subscribing to the cultural norm of youthfulness. There is a pattern of despair that permeates these women's lives and is reflected in dissatisfactions and worries about major aspects of their lives. It is suggested here that the Aged 2 women dealt with the crises created by the asynchrony between their inner biological and individual psychological dimensions by desiring to be younger than their chronological age. The following cases are illustrative.

A 45 year old white women who reported a subjective age of 55 years, responded that there was nothing good about being her age. She reported seven aging signs and symptoms and that the past five years were among the worst years of her life. This woman expressed worries about her income and health and reported only one satisfaction; her relationship with her children. She desired to be eighteen years of age. A 54 year old African American woman reported a subjective age of 80 years. Her health and income were grave sources of worry. She reported no satisfac- 
tions and seven aging signs and symptoms. This woman said that there was nothing good about being her age and that the past five years were among the worst in her life. She desired to be four years of age. Desiring to be a younger age offers these women the opportunity to use the cultural apparatus of chronological age as means of dealing with the contradictions and dialectical tensions in their lives.

Fortes (1984: 100) suggests that the "... cultural recognition of any stage of maturation takes note not only of biological signs but of achieved and expected capacities, skills and potentialities ...." It would appear that the women in Aged 2 group, while being culturally recognized as mothers, either do not have the achieved and expected capacities of parenthood or that they find the demands of this culturally recognized state of maturation more than they can reasonably handle. This contradiction is evidenced by the finding that the women desired to be younger than the age at which they had their first child. It appears that younger ages have symbolic importance for this group of women. For some, being younger means having someone take care of you. For example, one African American who desired to be one year old stated "I want to be back with my mother."

\section{CONCLUSION}

This research sought to answer the question: Do women who feel older and want to be younger than their chronological age differ on the variables of interest in this study from women who wish to be and feel their chronological age? The findings suggest that women who feel older and want to be younger than their chronological age perceive their lives to be stressful and contradictory. They worry about their health and income, have more aging signs and symptoms and are basically dissatisfied with the immediate contexts of their lives.

\section{NOTES}

* Revision of a paper presented at the 86th Annual American Anthropological Association Meetings, Chicago, IL, November 21, 1987. This project was supported in whole or in part by Division of Nursing, Public Health Service Grant NIH 5R21 NU00839 Awarded to the University of Michigan School of Nursing.

1 Although Kertzer and Keith briefly reviewed the psychological and sociological approaches to the study of adult development, their discussion tends to confuse the differences among them because they refer to life span development as "life course" and the life course perspective as age stratification.

${ }^{2}$ Stage theories of development are based upon Erikson's (1963) ontogenetic description of human development. According to Erikson (1963) developmental progression depends upon a series of chronologically ordered, essential developmetal tasks. Examples of the stage theories of development include Levinson et al. (1978) and Vaillant (1977). In fact 
Levinson et al. (1978) claim that their conception of the life structure is the generic human sequence.

In an excellent review of the major sociological and psychological theories of development, Rossi (1980) refers to stage theories as the "normative life crisis" model of development.

\section{REFERENCES CITED}

Alston, D. N. and N. Rose 1981 Perceptions of Middle-Aged Black Women as Related to Selected Background Factors. The Journal of General Psychology 104: 167-171.

Barnett, R. C. and G. K. Baruch 1978 Women in the Middle Years: A Critique of Research and Theory. Psychology of Women Quarterly 3: 187-197.

Bart, P. 1971 Depression in Middle-Aged Women. In Women in Sexist Society. V. Gornick and B. K. Moran, eds. Pp. 99-117. New York: Basic Books.

Brandes, S. 1985 Forty: The Age and the Symbol. Knoxville, Tenn.: University of Tennessee Press.

Brown, J. 1982 Cross-Cultural Perspectives on Middle-Aged Women. Current Anthropology 23: 143-156.

Brown, J. and V. Kerns 1985 In Her Prime: A New View of Middle-Aged Women. Massachusetts: Bergin \& Garvey.

Dannefer, D. 1984 Adult Development and Social Theory: A Paradigmatic Reappraisal. American Sociological Review 49: 100-116.

Datan, N., A. Antonovsky, and B. Maoz 1981 A Time to Reap: The Middle Age of Women in Five Isreali Subcultures. Baltimore: The Johns Hopkins Press.

Erikson, E. E. 1963 Childhood and Society (2nd ed.) New York: W. W. Norton.

Fortes, M. 1984 Age, Generation and Social Structure. In Age and Anthropological Theory. D. I. Kertzer and J. Keith, eds. Pp. 99-122. Ithaca, N.Y.: Cornell University Press.

Fry, C. L. 1980 Toward an Anthropology of Aging. In Aging in Culture and Society. C. L. Fry ed. Pp. 1-20. New York: J. F. Bergin.

Goody, J. 1976 Aging in Nonindustrial Societies. In Handbook of Aging and the Social Sciences. R. H. Binstock and E. Shanas, eds. Pp. 117-129. New York: Van Nostrand Reinhold Company.

Henry, J. 1973 Forty-Year-Old Jitters in Married Urban Women. In On Sham, Vulnerability, and Other Forms of Self-Destruction. Pp. 128-148. London: Penguin.

Kertzer, D. I. and J. Keith 1984 Age and Anthropological Theory. Ithaca, N.Y.: Cornell University Press.

Levinson, D. J., C. N. Darrow, E. B. Klein, M. H. Levinson, and B. McKee 1978 The Seasons in a Man's Life. New York: Knopf.

Neugarten, B. L. and N. Datan 1974 The Middle Years. In The American Handbook of Psyhciatry vol. 1 (2nd ed.). S. Arieti, ed. Pp. 592-608. New York: Basic Books.

Riegel, K. F. 1975 Toward a Dialectical Theory of Development. Human Development 18: 50-64.

Riegel, K. F. 1976 The Dialectics of Human Development. American Psychologist 31: $689-700$.

Riegel, K. F. 1979 Foundations of Dialectical Psychology. New York: Academic Press.

Riley, M. W. and R. P. Abeles 1982 Introduction: Life Course Perspectives. In Aging From Birth to Death: Sociotemporal Perspectives Vol. II. M. W. Riley, R. P. Abeles, and M. S. Teitelbaum, eds., Pp. 1-10. Boulder, CO: Westview Press.

Rossi, A. A. 1980 Aging and Parenthood in the Middle Years. In Life Span Development and Behavior Vol. 3. P. B. Baltes and O. B. Brim, eds., Pp. 137-205. New York: Academic Press. 
Rubin, L. B. 1979 Worlds of Pain: Life in Working Class Families. New York: Basic Books.

Simic, A. 1978 Introduction: Aging and the Aged in Cultural Perspective. In Life's Career - Aging: Cultural Variations on Growing Old. B. G. Myerhoff and A. Simic eds., Pp. 9-22. Beverley Hills: Sage Publications.

Steitz, J. A. 1981-82 The Female Lifecourse: Life Situations and Perceptions of Control. International Journal of Aging and Human Development 14: 195-204.

Vaillant, G. 1977 Adaptation to Life. Boston: Little Brown.

University of Michigan

School of Nursing and Department of Anthropology

Ann Arbor, MI 48109, U.S.A. 